\title{
Correlatos e variações na qualidade de vida dos servidores do enfrentamento ao sobrepeso numa IES pública na Cidade de Natal-RN
}

Correlates and variations in the quality of life of public servants to coping with overweight in a public IES in the City of Natal-RN

Correlaciones y variaciones en la calidad de vida de los servidores públicos con el afrontamiento del sobrepeso en una IES pública de la Ciudad de Natal-RN

\section{Resumo}

O tema sobrepeso e obesidade relacionada com a qualidade de vida (QdV) é um assunto de grande relevância para a humanidade, visto que propõem o resgate do valor das pessoas acometidas por essas patologias, assim como suas implicações na qualidade de vida desses indivíduos. Esse assunto pode proporcionar ações motivadoras, forças psíquicas e físicas dos sujeitos no que remete ao comportamento dos indivíduos para um melhor desempenho das atividades sociais e organizacionais. Nessa perspectiva, o presente estudo teve como objetivo identificar a percepção 
dos efeitos nos servidores públicos federais a respeito da sua participação no Projeto de Enfrentamento ao Sobrepeso e Obesidade. Tratou-se de uma pesquisa de campo, a qual, num primeiro momento se caracterizou como qualitativa e documental e num segundo momento do tipo quantitativa, descritivo e correlacional. Para coleta dos dados foram utilizados dois instrumentos com caracterização sociodemográfico, um instrumento de percepção do projeto e o outro o de QdV denominado SF-36, os quais foram aplicados a 20 servidores participantes. A análise dos dados foi realizada por meio da análise de conteúdo com base na metodologia de Bardin e do pacote estatístico SPSSWIN na versão 24.0 para tabular os dados e realizar as análises estatísticas. Os resultados revelaram que no aspecto qualitativo, apesar das propostas do projeto de QdV serem para a melhoria da vida dos seus participantes.

Palavras-chave: Qualidade de vida; Projeto de sobrepeso; Servidores públicos.

\begin{abstract}
The theme overweight and obesity related to quality of life (QoL) is a matter of great relevance to humanity, as they propose to rescue the value of people affected by these pathologies, as well as their implications for the QdL of these individuals. This subject can provide motivating actions, psychic and physical strength of the subjects, which refers to the behavior of individuals for a better performance of social and organizational activities. From this perspective, this study aimed to identify the perception of the effects on federal public servants regarding their participation in the Project to Combat Overweight and Obesity. It was field research, which, at first, was characterized as qualitative and documentary and, at a second moment, as quantitative, descriptive and correlational. For data collection, two instruments were used: Questionnaire, with the sociodemographic characterization and the perception of the project, and the SF-36, which were applied to 20 participating civil servants. Data analysis was performed using Bardin Content Analysis and SPSSWIN statistical package, in its version 24.0, to tabulate the data and perform descriptive statistical analyzes. The results revealed that in the qualitative aspect, despite the proposals of the project of QdL being for the improvement of the life of its participants.
\end{abstract}

Keywords: Quality of life; Overweight project; Public servants.

\title{
Resumen
}

El tema sobrepeso y obesidad relacionado con la calidad de vida (CdV) es un tema de gran relevancia para la humanidad, ya que proponen rescatar el valor de las personas afectadas por estas patologías, así como sus implicaciones para $\mathrm{CdV}$ de estos individuos. Esta asignatura puede aportar acciones motivadores, fuerza psíquica y física de los sujetos, que se refiere al comportamiento de los individuos para un mejor desempeño de las actividades sociales y organizacionales. Desde esta perspectiva, este estudio tuvo como objetivo identificar la percepción de los efectos en los servidores públicos federales en cuanto a su participación en el Proyecto de Lucha contra el Sobrepeso y la Obesidad. Se trató de una investigación de campo, que, en un primer momento, se caracterizó como cualitativa y documental y, en un segundo momento, como cuantitativa, descriptiva y correlacional. Para la recolección de datos se utilizaron dos instrumentos con caracterización sociodemográfica, uno de percepción del proyecto y otro el instrumento de CV denominado SF-36, los cuales se aplicaron a 20 servidores participantes. El análisis de los datos se realizó mediante análisis de contenido basado en la metodología Bardin y el paquete estadístico SPSSWIN en la versión 24.0 para tabular los datos y realizar análisis estadísticos descriptivos. Los resultados revelaron que en el aspecto cualitativo, apesar de que las propuestas de la proyecto of QdV son para la mejora de la vida de sus participantes.

Palabras clave: Calidad de vida; Proyecto de sobrepeso; Servidores públicos.

\section{Introdução}

O sobrepeso e a obesidade são problemas de saúde pública de grande relevância que vêm crescendo na população mundial e chamando a atenção dos pesquisadores para o estabelecimento de uma relação causal entre eles. Além disso, esses agravos são fatores de risco para doenças crônicas não transmissíveis (DCNTs), como doenças cardiovasculares, diabetes mellitus (DM) e hipertensão arterial, tendo como consequência a redução da qualidade de vida do indivíduo, a indisposição para o desenvolvimento das atividades laborais, visto que o excesso de peso repercute limitações motoras e até o risco aumentado de morte prematura, o que torna de suma relevância sua prevenção, sendo efetiva em qualquer nível, mesmo nas fases mais tardias da vida (Silva, Aguiar \& Fonseca, 2015).

A obesidade tem por definição o acúmulo anormal ou excessivo de gordura corporal em forma de tecido adiposo, podendo trazer riscos à saúde. É considerada um problema de saúde pública crescente, que tem se transformado em uma pandemia na sociedade ocidental (Venturini \& Ferreira, 2017). Segundo com a Organização Mundial de Saúde (2014), a obesidade é classificada de acordo com o cálculo do índice de massa corporal (IMC) (massa corporal/estatura2), sendo obeso 
grau I os que apresentam IMC de 30,0 a 34,9 kg/m2; obeso grau II com IMC de 35,0 a 39,9 kg/m2 e obeso grau III com IMC $\geq$ $40,0 \mathrm{~kg} / \mathrm{m} 2$.

Estudos realizados sobre o tema apontam uma etiologia complexa e multifatorial, compreendendo fatores genéticos, comportamentais, metabólicos e ambientais, estando diretamente associada ao risco de desenvolver doenças severas como diabetes mellitus, doenças cardiovasculares, dislipidemias, apneia obstrutiva do sono, osteoartrite e alguns tipos de neoplasias (Raiany et al., 2018).

De acordo com pesquisa realizada pela Vigilância de Fatores de Risco e Proteção para Doenças Crônicas por Inquérito Telefônico - Vigitel em 2017, 53,9\% dos brasileiros estão acima do peso ideal e destes, 18,9\% são obesos. Este estudo também revelou que o sobrepeso é maior entre os homens: $57,6 \%$ contra 50,8\% das mulheres. Contudo, a prevalência de obesidade é semelhante entre os gêneros.

Considerando o número de 5.300 servidores ativos na SUPRIMIDO., sendo cerca de 3.200 técnicos-administrativos em atividade e mais de 2.100 professores, estima-se que aproximadamente 2.856 estão com sobrepeso e 1.000 com obesidade exercem suas atividades laborais na instituição.

Dessa forma, esforços para melhorar esses índices podem ter importantes repercussões na qualidade de vida dos servidores e prevenir essas outras doenças. O tratamento da obesidade e do sobrepeso é complexo e muitas vezes abandonado, de forma que esforços individuais, profissionais e institucionais devam ser necessários para um resultado mais satisfatório. Neste contexto, acredita-se que uma abordagem multiprofissional e com grupos de ajuda mútua podem ser capazes de melhorar estas estatísticas (Dias et al., 2017).

Diante dessa realidade, destaca-se que estratégias de atendimento nutricional devem ser desenvolvidas para promover a perda de peso. Entre estas possíveis estratégias, o trabalho em grupo se faz eficaz por permitir à troca de vivências entre os participantes e dessa forma promover a sustentação mútua diante da fragilidade do participante com relação a seus problemas e angústias relacionadas ao quadro de obesidade (Abravanel, Socha, Sell, Angela \& Nesello, 2017).

Em 2016, a Diretoria de Atenção à Saúde do Servidor - DAS/PROGESP, instituiu o Projeto de Enfrentamento ao Sobrepeso e Obesidade na SUPRIMIDO, denominado inicialmente de PESO. Em 2019, esse projeto deu continuidade ao modelo inicial, com ajustes resultantes da avaliação da primeira turma de 2017, incorporando sugestões e correções de problemas, com o intuito de ajudar as pessoas com sobrepeso, obesidade e doenças relacionadas a essas condições, a adotarem hábitos de vida saudáveis, melhorando sua qualidade de vida.

As influências de caráter social, mental, histórico e político, onde o trabalho tem cunho central, possuem interferências na saúde dos indivíduos, na mesma direção em que a saúde influencia na realização do trabalho e em todos os aspectos da vida humana (Campos et al., 2017). Visto isso, nota-se que o trabalho é um grande influenciador na vida do ser humano e tem relação direta com a sua conduta fora desse ambiente. Então, intervenções em saúde e qualidade de vida, no âmbito pessoal e profissional, passam a ser elementos transformadores que promovem comportamentos saudáveis e que previnem enfermidades, capazes de conduzir as pessoas a uma qualidade de vida muito mais ampla do que simplesmente não estar doente (Grande, Botucatu \& Clementino, 2013).

Sendo assim, a qualidade de Vida, em seu sentido amplo, poderá ser compreendida como a harmonização de um conjunto de fatores que estão relacionados com a saúde física, psíquica, o nível de independência do sujeito, as relações sociais em casa, no trabalho e até mesmo a relação com o meio ambiente, ou seja, a sensação íntima de conforto, bem-estar ou felicidade no desempenho das funções (Maciel et al., 2016).

O Ministério da Saúde, em suas discussões aponta que nos últimos anos os projetos voltados à qualidade de vida vêm ganhando tamanha significância a ponto de aumentar a expectativa de vida dos indivíduos acometidos por doenças crônicas, assim como daqueles que apresentam grau de obesidade, condição que pode aumentar o risco de desenvolvimento de algumas 
dessas patologias. Essa é uma visão bastante ampla e que vem incentivando numerosas pesquisas na área da saúde, que engloba questões como, percepção da saúde, funções sociais, psicológicas e físicas assim como os dados a elas relacionados (Brand, Schuh, \& Gaya, 2013; Penteado \& Gastaldello, 2015).

No que se refere ao ambiente de trabalho, as instituições públicas, na sua forma mais objetiva, são consideradas órgãos direcionados à consecução do bem coletivo. Ao longo dos anos essas instituições, essas instituições não mudaram o seu objetivo, porém com algumas transformações teóricas, sofreram as influências impostas pela modernidade, dentre as quais podem ser citadas: sistematização das informações, inclusão de mídias digitais, realização das atividades fins através de servidores públicos concursados, dentre outras transformações vigentes atualmente (Carvalho \& Sampaio, 2010).

Por ser conhecido como um serviço ineficiente de cunho burocrático com baixo engajamento por parte dos gestores e do corpo operacional, ainda, há a ausência de foco nos resultados, o que leva a Administração Pública a adaptar-se lentamente às modificações impostas na contemporaneidade (Gomes \& Vieira, 2013).

$\mathrm{Na}$ atual conjuntura do mundo de trabalho, é observado que, cada vez mais o ambiente se encontrar envolvido com atitudes e comportamentos competitivos e complexos frente as exigências para uma melhor produtividade, portanto é imprescindível que as instituições públicas de ensino reconheçam a relação existente entre a atividade laboral, a saúde dos colaboradores, assim como os resultados organizacionais, investindo em programas de qualidade de vida dos seus servidores.

Entende-se que o processo de trabalho contemporâneo nas universidades influencia na saúde dos seus colaboradores podendo gerar sofrimento no âmbito físico, psíquico e social (Dias \& Rozendo, 2015). Com o intuito de preservar a saúde do trabalhador, no ano de 2009, através do decreto sob número 6.833, o Governo Federal lançou a Política de Assistência à Saúde e Segurança do Trabalho do Servidor Público Federal (PASS), fazendo com que a atenção à saúde do servidor federal fosse posta como objeto de grande relevância para as instituições, marcando assim uma conquista após uma longa luta da categoria por políticas públicas voltadas para esse campo. Dessa forma, diversas instituições federais aderiram ao PASS, seguindo seus princípios e consequentemente o seu regimento (Coelho, 2016).

Nesse sentido este estudo tem como questão norteadora identificar os reflexos de um projeto de qualidade de vida dos participantes, os quais são servidores de uma instituição pública. A pesquisa relevante, visto que será permissível uma análise da metodologia utilizada do programa, como também a implantação de meios para combater a evasão do projeto de Enfrentamento ao Sobrepeso e à Obesidade para Servidores da SUPRIMIDO., assim como motivar os servidores a buscarem saúde e qualidade de vida dentro e fora do ambiente de trabalho.

Segundo Pasternak (2016), saúde é a junção completa bem-estar físico, mental e social, o que significa que não se resume, apenas, a ausência de doença, visto que é possível a presença simultânea de saúde e de certas patologias, em fase inicial, as quais se comportam de maneira assintomática ainda, e não deixa o paciente com limitações funcionais. Ou seja, A saúde está indiscutivelmente relacionada com o bem-estar e a doença como um desequilíbrio, ambos com repercussões e implicações em diferentes dimensões, física, psicológica, cultural, emocional, ambiental, econômica e espiritual (Araújo, 2006).

Essa concepção é bastante ampla, o que vem a envolver também aspectos socioeconômicos, além da saúde física e mental. Alvo de inúmeras críticas, essa definição ainda é discutida, visto que um estado de completo bem-estar transparece que a saúde ideal seja algo inatingível (Corrêa et al., 2017).

Para a maioria das pessoas estar em condições de boa saúde é estar apto para a realização das suas atividades diárias. Numa perspectiva ecológica, a saúde é considerada um elemento fundamental na evolução das espécies vivas, o que não é suficiente, apenas, ter seus órgãos em funcionamento, é essencial que o indivíduo esteja em pleno vigor para

desenvolver suas obrigações pessoais, assim como ter disposição para enfrentar as demandas rotineiras do seu ambiente laboral com o mínimo de absenteísmo possível, que tenha os momentos de relaxamento e calma, assim como 
expectativas de futuro, desejo e equilíbrio entre o ambiente em que vive e os seus ideais. (Oliveira, 2009).

Segundo Cortina e Llopies (1988, p. 143), a saúde é uma interligação entre o ambiente, stress, biologia, hábitos de vida, e sistema de Saúde. É de extrema necessidade considerar que saúde também implica a capacidade de adaptação do indivíduo às alterações contínuas do meio em que vive, uma vez que todos os aspectos da saúde estão inter-relacionados e são interdependentes (Gaspar, 2006).

A mesma opinião é manifestada por Costa e López (1998), ao referirem que o novo conceito de saúde implica dar poder ao indivíduo para que este se desenvolva tanto na vida social como na vida profissional e tenha capacidade de influenciar, positivamente, os ambientes sociais e laborais, assim tornando-os capazes para realizar tomadas de decisões (Gaspar, 2006).

Como parte integrante da $\mathrm{QV}$, a qualidade de vida no trabalho é um termo bastante utilizado nos últimos vinte anos, visto que é cada vez mais evidente a procura por equilíbrio entre qualidade de vida e carreira profissional. Visto isso, grandes empresas já estão se adaptando a essa nova demanda realizando investimentos em ações de promoção da qualidade de vida dos seus colaboradores (Souza, 2015). Porém isto não significa que as empresas de pequeno e médio porte não irão se repaginar diante dessa nova metodologia, pelo contrário, para fazer frente às grandes empresas e não perder seus melhores talentos, esses estabelecimentos devem descobrir o que representa qualidade de vida para os seus empregados através de diálogos abertos e repassando para eles o poder da opinião, a fim de levantar as necessidades desses indivíduos e essa formar criar programas de incentivo ao bem estar (Rueda, Serenini, \& Meireles, 2014).

É importante salientar que, não se deve esquecer de que a QV é algo extremamente subjetivo, ou seja, para uns significa passar mais tempo com a família, para outros é poder se dedicar ao trabalho ou conseguir manter uma alimentação saudável, são inúmeras as situações que representam qualidade de vida para as pessoas e certamente dentro das empresas não seria diferente, pois nelas são encontrados interesses distintos, por parte dos empregados, e não dá para fazer uma ação para atender cada um deles, então conhecer o que pode ter de comum entre todos da equipe pode representar uma boa solução para esta situação (Sousa \& Fernandes, 2014).

A qualidade de vida, seja no trabalho seja na vida pessoal, tem reflexo direto na saúde física e emocional do ser humano e consequentemente na produtividade das suas atividades laborais, motivo pelo qual as grandes empresas já estão investindo alto para a melhoria contínua da saúde dos seus empregados, portanto esses investimentos devem ser contínuos, pois o mundo de trabalho está inserido em movimentos para responder às pressões impostas nas demandas diárias, as quais requerem menos tempo e mais qualidade. Afinal, para manter a continuidade produtiva, faz-se a necessidade de profissionais saudáveis (Parise \& Soler, 2016).

Logo, para alcançar os resultados mais esperados pelas empresas, foram criados os movimentos de Programa de Qualidade de Vida no Trabalho (PQVT), o qual se deu na década de 50 com a abordagem sociotécnica, porém foi a partir dos anos 60 que os líderes sindicais, empresários e governantes tomaram a iniciativa da implantação dos PQVT nos ambientes laborais com o intuito de minimizar os efeitos negativos do trabalho na vida dos trabalhadores (Chiavenato, 1983).

Segundo Carvalho, et al. (2013), na conjuntura atual do mercado, o principal objetivo da inserção dos PQVT é repaginar os ambientes laborais com o intuito de proporcionar ao trabalhador suas necessidades básicas, assim, de forma satisfatória, atingir os resultados da empresa. Nesse contexto a qualidade de vida no trabalho proporciona uma relação de reciprocidade entre emprego e empregador, ou seja, à medida que a organização tem a ação de promover a satisfação no trabalho dos seus colaboradores, esses terão a reação de efetuar uma melhoria produtiva.

Então, a partir desse ponto de vista, diversas instituições vêm aderindo aos projetos de QVT, visto que os benefícios desses projetos são inúmeros, uma vez que trazem para o ambiente organizacional, práticas que ajudam no desenvolvimento de suas atividades e dos seus profissionais. Um dos exemplos disso são os programas de ginástica laboral e ergonomia que trazem 
atividades físicas para o ambiente de trabalho e orientam os profissionais a terem uma postura corporal mais adequada (Melo, 2016).

Outro programa importante são os de treinamento e desenvolvimento; para capacitação dos colaboradores por meio de cursos, treinamentos, palestras e workshops, assim como realização de exames periódicos com o intuito de oferecer a promoção da saúde. Visto isto, esta valorização mostra o comprometimento das empresas em fazer com que seus profissionais possam desenvolver seus talentos e habilidades de forma progressiva e com isso, aumentar sua produtividade e, também o seu engajamento e motivação (Costa, 2014).

Esta pesquisa desenvolverá seu estudo em um dos Programas de QVT regulamentado através da Resolução n012/2017 do Conselho de Administração (CONSAD)que regulamenta o "Viver em Harmonia", que está vinculado à Coordenadoria de Qualidade de Vida no Trabalho (CQVT) da Pró-Reitoria de Gestão de Pessoas (PROGESP), cuja competência é coordenar, executar e acompanhar ações de promoção à satisfação, reconhecimento sócio profissional, relações interpessoais harmoniosas, promover um ambiente laboral saudável com equilíbrio entre o trabalho e a vida pessoal.

A participação nesses programas está restrita a docentes e técnicos administrativos em educação da SUPRIMIDO., desde que haja o consentimento da sua chefia imediata. E como meio de incentivo para a participação do máximo de servidores, esta resolução, no seu capítulo III, explicita que nos casos em que o horário da atividade escolhida pelo servidor coincida com o horário de trabalho, para aqueles com jornada acima de 06(seis) horas ou com jornada estabelecida em normatização específica, será devido o abono pela chefia imediata de no máximo 05 (cinco) horas semanais no registro de frequência. Com isso, este estudo tem como principal objetivo, verificar o quanto o funcionário que participara do programa de QVT na instituição é capaz de reconhecer a mudança na qualidade de vida e as funções multidimensionais desta na sua vida e no trabalho.

\section{Metodologia}

\section{Modelo da Pesquisa}

Trata-se de um estudo descritivo, exploratório e correlacional, de abordagem quantitativa envolvendo funcionários de uma IES na cidade de Natal, RN; para isso utilizou-se à abordagem não probabilística em bola de neve, fazendo-se necessário avaliar o 'n' amostral mais adequado para a realização do estudo.

Através do pacote estatístico GPower 3.1 verificou-se a qualidade da amostra no estudo. Esse software é destinado ao cálculo do poder estatístico, tendo como base, não apenas o 'n' necessário para a pesquisa, mas, também, o tipo de cálculo a ser realizado no estudo em questão (Faul, Erdfelder, Lang \& Buchner, 2007).

\section{Participantes da Pesquisa}

Para a amostra coletada, considerando uma probabilidade de $95 \%(\mathrm{p}<0,05)$, magnitude do efeito amostral $(\mathrm{r} \geq 0,50)$ e um padrão de poder hipotético ( $\pi \geq 0,80$ ), observou-se que, a partir desses critérios, a referida amostra (20 sujeitos), mostrouse adequada para a realização do objetivo de estudo, pois, os indicadores da qualidade amostral estiveram dentro do exigido ( $t$ $\geq 1,98 ; \pi=0,91 ; \mathrm{p}<0,05)$.

Considerou-se os servidores da própria instituição que atuam nas diversas áreas da mesma e que foram inscritos de forma efetiva no programa de apoio destinado ao enfrentamento do sobrepeso e que frequentaram pelo menos oito encontros do programa. Bem como, aqueles que a partir da avaliação do IMC, apresentavam índice de sobrepeso.

\section{Instrumentos para coleta de dados}

Além dos dados sociodemográficos, os participantes responderam o seguinte instrumento: 
- Medical Outcomes Study36 (SF-36), esse instrumento objetivou mensurar a Qualidade de Vida, apresentando consistência interna satisfatória na pesquisa, o que resultou em um recurso de fácil administração. Esse instrumento é constituído por um questionário multidimensional, formado por 36 itens incluindo oito componentes: Capacidade funcional (com dez itens); Aspectos físicos (quatro itens); Dor (dois itens); Estado geral de saúde (cinco itens); Vitalidade (quatro itens); Aspectos sociais (dois itens); Aspectos emocionais (três itens); Saúde mental (cinco itens); e mais uma questão para a avaliação comparativa do estado geral de saúde atual e a de um ano atrás. Os componentes podem ser agrupados em dois sumários, o físico (Capacidade funcional, Aspectos físicos, Dor e Estado geral de saúde) e o mental (Saúde mental, Aspectos emocionais, Aspectos sociais e Vitalidade).

\section{Procedimento de coleta e análise de dados}

Após aprovação pelo Comitê de Ética, os instrumentos foram encaminhados aos respondentes através de um formulário eletrônico disponível online na página do GoogleForms. Ao solicitar a participação dos servidores, foi informado a esses respondentes que o objetivo do estudo seria o de identificar a percepção do efeito nos servidores públicos federais da sua participação no Projeto de Enfrentamento ao Sobrepeso e Obesidade. Aos servidores foi solicitada sua participação voluntária, com assinatura virtual do Termo de Consentimento Livre e Esclarecido (TCLE).

As pessoas que se mostraram interessadas e deram o seu consentimento em participar para fazer parte da amostra do estudo, foram esclarecidas que as suas respostas seriam pessoais e sem interferência do coordenador da pesquisa, assim, ao responder o instrumento, não haveria respostas certas ou erradas e elas seriam tratadas de acordo com o que o sujeito pensou ao ler as questões apresentadas e indicou a sua resposta no instrumento apresentado, assim como solicitação de análise dos seus prontuários.

Também foi assegurado o anonimato das respostas, bem como, que as questões respondidas foram tratadas em seu conjunto de resposta e não na particularidade da resposta de cada sujeito. Apesar de se encontrar as instruções necessárias para que o questionário pudesse ser respondido, o pesquisador (com experiência prévia na pesquisa) colocou-se à disposição para esclarecimento sobre as dúvidas que surgiram; um tempo médio de 20 minutos foi suficiente para que a atividade pudesse ser concluída.

Quanto a categorização dos dados, utilizou-se o Pacote estatístico SPSSWIN, em sua versão 21.0 para tabular os dados e realizar as seguintes análises: estatísticas descritivas (média, desvio padrão, mediana), Correlação de Pearson, Teste de t de Student, Alfa de Crombach e Qui-quadrado.

\section{Resultados e Discussão}

Com a coleta de dados finalizadas, realizaram-se as análises referentes a qualidade da amostra para a referida pesquisa: verificou-se a existência da multicolinearidade entre as variáveis, as quais, não apresentaram correlações acima do permitido (isto é, $r \geq 0,90$ ), observando correlações ariando de $-0,03$ a 0,69 . Este resultado permite afirmar que as variáveis não apresentaram um alto grau de correlação, permitindo gerar modelos explicativos com baixo erro de medida; avaliou-se a possível tendência de não resposta, utilizando o procedimento de Armstrong e Overton (1977), através do qual avaliaram-se as diferenças entre os primeiros $(\mathrm{n} 1=5)$ e os últimos respondentes $(\mathrm{n} 2=5)$ usando o teste de Levene, destinado a verificação da igualdade de variâncias e, em seguida, um teste-t para testar a igualdade de meios (Dancey \& Reidy, 2006). Com isso, não houve resultado significativo a um nível de $5 \%$ entre os escores médios dos construtos referentes aos dois grupos, com um indicador $t \leq 1,96$, constatando assim, que o viés de não resposta não é um problema significativo.

A amostra do estudo foi composta por 20 servidores, sendo, todos funcionários de diversos setores da SUPRIMIDO na cidade de Natal-RN. O gênero feminino predominou na amostra dos participantes (70\%), corroborando os estudos de (Lasta 
et al., 2018), os quais revelam que o público do sexo feminino tem maiores intenções de envelhecer de forma saudável, o que deve incluir boa saúde física e mental, ser independente, ter um convívio social, boa estrutura familiar e situação financeira. Com essas práticas os efeitos do envelhecimento podem ser minimizados, melhorando a força, a massa muscular, a flexibilidade, o sistema circulatório, o aumento da capacidade cardiorrespiratória, a glicemia controlada, IMC adequado e um melhor equilíbrio, diminuindo a incidência de quedas, além da melhora da autoestima e autoconfiança.

No que se refere a idade, esta variou de 21 a 30 anos (10\%), 31 a $40(35 \%), 41$ a $50(25 \%), 51$ a $60(25 \%)$ e 61 a 70 (5\%), demonstrando que a maior incidência de procura por esses projetos se configura com profissionais da fase adultez final, onde as pessoas tendem a traçar suas metas (Adriana \& Souza, 2017).

Caracterizando ainda os dados sociodemográficos, pode ser visto também que $80 \%$ dos entrevistados eram casados, o que presume serem pessoas com maiores demandas diárias e consequentemente com maiores responsabilidades, o que reflete em um menor tempo para o cuidado pessoal. Quanto ao nível de escolaridade dos respondentes, 50\% possuem o nível de graduação, o que indica que não é necessário, apenas, ter um nível de escolaridade superior para se ter conhecimento de hábitos de vida saudáveis, faz-se necessária também a autorreflexão da conduta de vida. Do total de entrevistados, $70 \%$ têm um tempo de atuação acima de 10 anos, o que comprova uma vida com hábitos sedentários e uma possível alimentação inadequada.

Verificado a normalidade da amostra e seguro de que a escala utilizada tem sido administrada em inúmeros estudos com distintas amostras, as quais vem revelando indicadores psicométricos confiáveis, certo da sua qualidade, optou-se em avaliar a consistência interna da escala em questão, o SF-36. Partiu-se do pressuposto teórico de que tal medida se organiza fatorialmente em oito dimensões, as quais tanto tem sido consistente ao longo do tempo em diversas pesquisas a qualidade da sua mensuração, bem como, atende axiomaticamente, a perspectiva conceitual referente a qualidade de vida a ser avaliada nos respondentes desta pesquisa (Laguardia, Campos, Flor \& Portugal, 2016).

Por ser uma amostra com menos de 30 sujeitos e ter participante de um grupo muito específico para o resgate da qualidade de vida de funcionário, viu-se a necessidade de verificar a qualidade psicométrica do SF-36, para isso, utilizou-se o cálculo do Lambda 2 de Guttman ( $\lambda 2$ 2). De acordo com Formiga, Souza, Costa, Fleury e Melo (2015, p. 94-95) a intenção no uso desse coeficiente se deve ao fato de encontrar alguns estudos, que este indicador expressa uma melhor estimativa de confiabilidade quando o instrumento avaliado é composto por poucos itens que formam s fatores ou uma amostra pequena. Sendo assim, este indicador deve assumir um escore acima de 0,70 para se afirmar a qualidade da medida aplicada. De acordo com Pasquali (2011), tal escore se mantendo neste critério, significa dizer que os itens são homogêneos em sua mensuração, os quais, seguem uma direção de medida na mesma variância, o que, caracteriza segurança e fidelidade da medida sobre o fenômeno avaliado.

Tendo assumido esse critério psicométrico, para à avaliação da escala SF-36 e suas dimensões, observou-se o seguinte resultado: com exceção da dimensão AS (Aspectos Sociais), a qual, não apresentou escore maior do que 0,70, sendo assim, não confiável para sua avaliação na referida amostra. As demais, dimensões são fidedignas, pois, estiveram acima do indicador psicométrico exigido (>0,70) (ver tabela 1$)$.

Ainda na mesma Tabela 1, utilizou-se também, da avaliação da correlação intraclasse; observou-se que o ICC esteve cima de 0,70. A dimensão AS não apresentou ICC adequado, com isso, sugere-se exclui-la da avaliação nos cálculos inferenciais, até porque, tanto neste indicador quanto no Lambda de Guttman, os resultados não foram significativos, apresentando uma probabilidade (p-valor) maior do que 0,05 . 
Tabela 1: Escores dos alfas de Cronbach $(\alpha)$ do SF-36 e suas dimensões.

\begin{tabular}{|c|c|c|c|c|c|}
\hline \multirow[b]{2}{*}{ Construto/Dimensão } & \multicolumn{3}{|c|}{ Lambda de Guttman } & \multirow[b]{2}{*}{$\begin{array}{c}\text { ICC } \\
(\mathrm{IC} 95 \%)\end{array}$} & \multirow[b]{2}{*}{ p-valor } \\
\hline & $\alpha_{\text {geral }}$ & $\mathrm{V} \lambda_{\text {Item excluído }}$ & $\begin{array}{c}\mathrm{F} \\
\text { Friedman }\end{array}$ & & \\
\hline QVsf-36 & 0,75 & $0,69-0,75$ & 23,04 & $\begin{array}{c}0,75 \\
(0,69-0,73)\end{array}$ & 0,01 \\
\hline $\mathrm{CF}$ & 0,94 & $0,91-0,93$ & 16,63 & $\begin{array}{c}0,92 \\
(0,87-0,97)\end{array}$ & 0,01 \\
\hline $\mathrm{AF}$ & 0,73 & $0,69-0,75$ & 5,71 & $\begin{array}{c}0,73 \\
(0,70-0,88)\end{array}$ & 0,01 \\
\hline $\mathrm{AE}$ & 0,73 & $0,69-0,75$ & 6,82 & $\begin{array}{c}0,70 \\
(0,69-0,77)\end{array}$ & 0,01 \\
\hline AS & 0,50 & $0,42-0,55$ & 1,30 & $\begin{array}{c}0,56 \\
(0,49-0,60)\end{array}$ & 0,59 \\
\hline DOR & 0,84 & $0,81-0,89$ & 12,84 & $\begin{array}{c}0,84 \\
(0,79-0,94)\end{array}$ & 0,01 \\
\hline VIT & 0,78 & $0,71-0,82$ & 11,98 & $\begin{array}{c}0,77 \\
(0,75-0,85)\end{array}$ & 0,01 \\
\hline $\mathrm{SM}$ & 0,74 & $0,69-0,73$ & 30,31 & $\begin{array}{c}0,71 \\
(0,69-0,86)\end{array}$ & 0,01 \\
\hline EGS & 0,76 & $0,72-0,79$ & 3,82 & $\begin{array}{c}0,71 \\
(0,69-0,83)\end{array}$ & 0,01 \\
\hline
\end{tabular}

Notas: QVsf-36 = Qualidade de Vida [pontuação total], $\mathrm{CF}=$ capacidade funcional, $\mathrm{AF}=$ aspectos físicos, $\mathrm{AE}=$ aspectos emocionais, $\mathrm{AS}=$ aspectos sociais, $\mathrm{DOR}=$ dor, VIT = vitalidade, $\mathrm{SM}=$ saúde mental, EGS estado geral de saúde; $\mathrm{V} \alpha=$ Variação alfa quando o item for excluído; F = Teste de Friedman; ICC = Correlação intraclasse.

Fonte: Autores.

Com base nestes resultados e sabendo da qualidade psicométrica da medida em questão, realizou-se o cálculo de correlação de Pearson entre as variáveis mensuradas a fim de atender ao objetivo proposto. Inicialmente, efetuou-se entre a medida da qualidade de vida e suas dimensões com as variáveis sociodemográficas. Em relação a variável qualidade de vida geral (pontuação total da escala), houve uma correlação positiva apenas com a escolaridade.

No que se refere as dimensões do construto da qualidade de vida e sua relação com as variáveis sociodemográficas, apenas o aspecto físico (AF) correlacionou-se negativamente, com o sexo; saúde mental (SM) também, relacionou-se negativamente, com escolaridade, com cargo e tempo de serviço; na mesma direção de força (negativa) o estado geral da saúde (EGS) correlacionou-se com escolaridade.

No que se refere as dimensões do construto da qualidade de vida e sua relação com as variáveis sociodemográficas, apenas o aspecto físico (AF) correlacionou-se negativamente, com o sexo; saúde mental (SM) também, relacionou-se negativamente, com escolaridade, com cargo e tempo de serviço; na mesma direção de força (negativa) o estado geral da saúde (EGS) correlacionou-se com escolaridade. 
Tabela 2: Escores correlacionais do SF-36 e suas dimensões com as variáveis Sociodemográficas.

\begin{tabular}{lcccccc}
\hline Dimensões & Sexo & Idade & Escolaridade & Cargo & $\begin{array}{c}\text { Tempo } \\
\text { de Serviço }\end{array}$ & $\begin{array}{c}\text { Estado } \\
\text { Civil }\end{array}$ \\
\hline QVsf-36 & 0,12 & 0,14 & $0,52^{*}$ & $-0,14$ & $-0,10$ & $-0,26$ \\
CF & 0,33 & 0,15 & 0,04 & 0,29 & 0,16 & $-0,01$ \\
AF & $-0,52 *$ & 0,05 & 0,11 & 0,21 & 0,30 & $-0,14$ \\
AE & $-0,23$ & 0,12 & 0,01 & 0,01 & 0,08 & 0,17 \\
DOR & 0,15 & 0,14 & 0,36 & 0,14 & 0,27 & 0,14 \\
VIT & $-0,31$ & 0,20 & 0,07 & 0,03 & 0,29 & $-0,07$ \\
SM & 0,04 & $-0,31$ & $-0,53^{*}$ & $-0,51 *$ & $-0,51 *$ & $-0,19$ \\
EGS & $-0,18$ & 0,07 & $-0,54^{*}$ & $-0,36$ & 0,36 & $-0,05$ \\
\hline
\end{tabular}

Notas: QVsf-36 = Qualidade de Vida [pontuação total], CF = capacidade funcional, $\mathrm{AF}=$ aspectos físicos, $\mathrm{AE}=$ aspectos emocionais, $\mathrm{AS}=$ aspectos sociais, $\mathrm{DOR}=$ dor, $\mathrm{VIT}=$ vitalidade, $\mathrm{SM}=$ saúde mental, EGS $=$ estado geral de saúde. $*$ p $<0,05$.

Fonte: Autores.

A partir dessas análises efetuou-se uma correlação de Pearson, associando as variáveis da qualidade de vida com as variáveis sobre percepção que o respondente tem sobre a sua saúde (por exemplo, em geral você diria que sua saúde é; comparada há um ano atrás, como você descreve o seu estado geral de saúde). Com isso, na Tabela 3, observou-se que a variável da percepção que o respondente tem sobre a sua saúde relacionou-se apenas com a dimensão geral qualidade vida (QVsf-36) e capacidade funcional (CF); no que diz respeito a variável Comparada há um ano atrás, como descreve o seu estado geral de saúde atual, esta relacionou-se, positivamente, somente com capacidade funcional (CF) e, negativamente, com saúde mental (SM).

Tabela 3: Escores intra-correlacionais do SF-36 e suas dimensões.

\begin{tabular}{ccc}
\hline Dimensões & $\begin{array}{c}\text { Em geral você diria que a } \\
\text { sua saúde é }\end{array}$ & $\begin{array}{c}\text { Comparada há um ano atrás, como } \\
\text { descreve o seu estado geral de saúde } \\
\text { atual. }\end{array}$ \\
\hline QVsf-36 & $0,46^{*}$ & 0,03 \\
CF & $0,41^{*}$ & $0,48^{*}$ \\
AF & 0,11 & $-0,04$ \\
AE & $-0,16$ & $-0,24$ \\
DOR & 0,14 & 0,28 \\
VIT & $-0,13$ & $-0,38$ \\
SM & 0,01 & $-0,45^{*}$ \\
EGS & 0,05 & $-0,19$ \\
\hline
\end{tabular}

Notas: QVsf-36 = Qualidade de Vida [pontuação total], CF = capacidade funcional, AF = aspectos físicos, $\mathrm{AE}=$ aspectos emocionais, $\mathrm{AS}=$ aspectos sociais, $\mathrm{DOR}=$ dor, $\mathrm{VIT}=$ vitalidade, $\mathrm{SM}=$ saúde mental, $\mathrm{EGS}=$ estado geral de saúde. $* \mathrm{p}<0,05$.

Fonte: Autores.

A partir dessas relações, optou-se em realizar o cálculo do qui-quadrado para o percentual de concordância entre as variáveis. Sendo assim, para verificar os efeitos destas, as variáveis independentes foram categorizadas tercis, distribuídas nos seguintes níveis $(1=$ baixo, 2 = moderado e 3 = alto). Com base nesta distribuição, serão apresentados os resultados relativos a cada variável independente; na Tabela 4 pode-se observar que as mulheres tiveram maiores percentuais na moderada qualidade de vida. 
Tabela 4: Frequência em percentagem na QV em função do sexo.

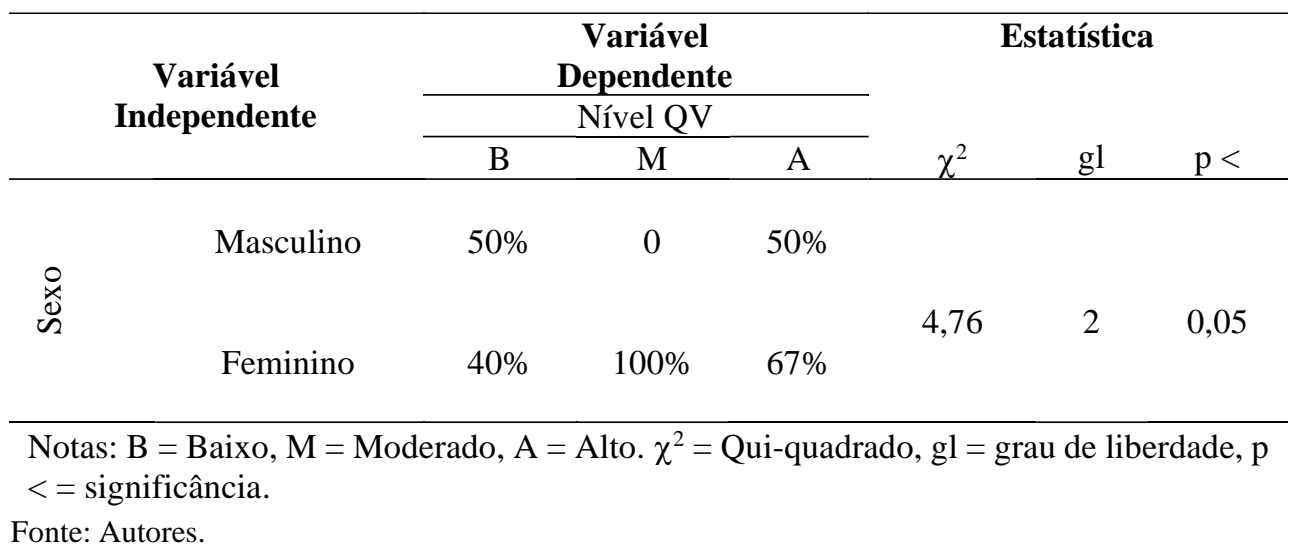

Considerando a idade, esta foi distribuída em cinco níveis etários; na tabela 5, pode-se observar que, apesar de existir um alto percentual relativo a alta qualidade de vida em alguns níveis etários, chama-se atenção para a faixa de 61 a 70 anos, tendo $100 \%$ destes respondentes numa maior qualidade de vida; mesmo assim, esses resultados não foram significativos.

Esta pesquisa teve como objetivo avaliar a qualidade de vida e saúde na percepção de servidores públicos que participam de um projeto de enfrentamento ao sobrepeso oferecido por uma instituição pública de ensino superior no Rio Grande do Norte. Os resultados apresentados procuraram contribuir, seja na sua forma avaliativa através do método qualitativo e quantitativo, avaliar a qualidade de vida em servidores públicos sobre um projeto de enfrentamento ao sobrepeso.

Sendo assim, no que se refere aos resultados qualitativos, pode ser visto que a caracterização sociodemográfica dos profissionais participantes da pesquisa confirmou os resultados dos estudos realizados por Cura (2012), Sawada e Galvão (2013) e Carvalho et al., (2013), o que reafirma a importância da inserção de projetos de qualidade de vida nos ambientes laborais.

Para Meirlles (2014), a relevância dos projetos de qualidade de vida para os trabalhadores é reflexo do espaço que esses projetos vêm ganhando na Administração Pública e tem como objetivo central melhorar as condições de trabalho para os funcionários da instituição e, com isso, aumentar a produtividade.

Então, visando uma melhoria da qualidade de vida dos seus colaboradores, a SUPRIMIDO desenvolve projetos de QV, os quais motivam os servidores dessa instituição a participarem. E um desses projetos é o Enfrentamento ao Sobrepeso e à Obesidade onde a grande maioria dos participantes relata o sobrepeso como causa principal da procura.

Esses projetos se mostram com um meio de divulgação das inscrições, bastante eficaz, pois a grande maioria os participantes expões que ficaram sabendo da existência através do SIGRH.

Do ponto de vista da saúde do trabalhador e da satisfação com o trabalho, o tempo de serviço é uma variável importante, uma vez que quanto maior o tempo de atuação em uma instituição, maior a exposição dos profissionais aos fatores de riscos físicos, biológicos, ergonômico, psicossocial e químico. Nesse estudo há profissionais com mais de 10 anos de atuação na instituição que participaram do estudo

Porém Guido (2014), referiu que não é possível afirmar que a experiência profissional diminua a vulnerabilidade dos trabalhadores a doenças ocupacionais, como estresse relata em seu estudo que, quanto maior o tempo de atuação menor o estresse nas atividades laborais. Neste mesmo estudo observou-se também que, a maioria dos trabalhadores era do sexo feminino, com estado civil casado e nível de escolaridade de graduação.

A partir desses resultados, pode-se destacar que na concepção qualitativa, os entrevistados se mostram motivados com o projeto, porém há a necessidade de uma metodologia mais incisiva para que fique claro para os participantes o real objetivo 
do projeto de qualidade de vida em estudo, visto que em um conjunto de 20 servidores do estudo emergiram três categorias de respostas e dessas sugiram 15 subcategorias, as quais apresentam em sua maioria que o indivíduo tem um pensamento duvidoso com relação às atitudes de novos hábitos de vida e qualidade de vida, ou seja, o indivíduo ainda é confuso, e deve ser trabalhada a área psicológica desse público para que eles pensem de forma correta, a ponto desses hábitos serem praticados a longo prazo.

Em relação aos resultados quantitativos, por ser um estudo pioneiro na presente amostra e contexto social e institucional de pesquisa, procurou-se realizar uma análise de consistência das medidas utilizadas; estes garantiram o uso da escala de qualidade de vida em sua pontuação total, bem como, em seus respectivos fatores, com exceção do fator AS (Aspetos Sociais), o qual, não apenas não foi significativo, bem como, não revelou uma alfa acima de 0,70 . Esta condição permitiu ao autor, não considerar esse fator na avaliação dos escores para os respondentes.

Com isso, é preciso destacar que diferente do processo de verificação psicométrica da escala, não apenas é proposto a exclusão do fator, mas, também, é possível refletir que, não alcançando o indicador proposto, pode-se afirmar que este fator (Aspectos Sociais), neste grupo de respondentes, não houve possibilidade de avaliação tais aspectos.

Destacou-se também, os valores do coeficiente e intervalos intraclasse, estes, destinados à avaliação do limite de confiança relativo a realização de futuros estudos com amostras de características semelhantes as coletadas para este capítulo, os quais, confirma que apenas o fator AS não foi adequado, mas, os demais fatores estiveram no intervalo aceitável.

No que se refere as relações da QV e seus fatores relacionados as variáveis sociodemográficas, poucas relações foram significativas com algumas se relacionando negativamente (por exemplo, AF, SM e EGS), pois, esperava-se que alguns desses fatores fossem relacionados positivamente, já que o programa de enfrentamento tem como proposta a melhoria na vida e na saúde, especialmente, a saúde mental (a EGS na QV). Nestas relações, apenas a pontuação total da qualidade de vida (QV) relacionou-se, significativamente, com a escolaridade.

Outro resultado que merece ser destacado é quanto a relação da QV e seus fatores com as variáveis da percepção da saúde (por exemplo, autoavaliação da saúde atual e comparação da saúde atual em relação a ano anterior); com isso, houve a relação positiva da QV e CF com autoavaliação da saúde atual e com a variável comparação da saúde atual em relação ao ano anterior, ter sido apenas com a CF positivo e significativamente e com SM correlacionando negativamente, é possível destacar que pouco foi alterado o sentido e significado da saúde destes profissionais.

Esse detalhe pode ser destacado quando se avaliou CF, pois, o escore correlacional foi maior para a auto informação de que a sua vida estava melhor a um ano anterior ao programa. Com isso, frente a esses resultados, é preciso certa parcimônia na avaliação do benefício desse programa do enfrentamento ao sobrepeso, pois, provavelmente, tal abordagem do programa, considerando esses resultados, poderá ser bastante tímido.

A partir destes resultados, avaliou-se o percentual entre as variáveis sociodemográficas e o QV e suas dimensões. Observou que as mulheres apresentaram maior percentual na categoria moderada para alta, porém, é preciso destacar que a amostra (n) nestes resultados não são significativas (pois, varia de 1 a 3 mulheres), sendo assim, é preciso maior cuidado quando se refletir sobre o êxito do programa de enfrentamento.

Outra reflexão que merece ser destacada é quanto a influência da idade em função da QV e seus fatores; apenas o AF (Aspectos Físicos) apresentou resultado significativo, chamando atenção para a faixa etária de 61 a 70 anos apresentando maior percentual na alta categoria de AF; mas, neste resultado é preciso também, um cuidado na sua avaliação significativa, pois, observou-se que em outras faixas etárias ( 31 a 40 anos e 41 a 50 anos e 51 a 60 anos) também tiveram percentuais acima de 50\%. Resultados esses, que merecem maior atenção no que se refere as questões teóricas e empíricas. 
Por fim, um resultado que poderia contribuir para este estudo e a reflexão do programa de enfrentamento, refere-se à avaliação do pareamento entre os fatores do QV; nestes resultados, pode-se destacar, hierarquicamente, os fatores da QV que merecem prioridade quando na execução das atividades do programa.

Nesta análise, nota-se que seria de grande utilidade iniciar o programa pela CV, seguido de SM e assim, sucessivamente. Desta maneira, sugere-se que outros instrumentos podem ser utilizados para a comparação da QV de forma mais reduzida, já que no conjunto fatorial deste instrumento é possível reduzi-los em fatores psicológicos e comportamentais, o que facilitaria muito a avaliação geral da QV (Rufine, Formiga. \& Melo, 2013).

\section{Conclusão}

O sobrepeso e a obesidade tornaram-se problema de saúde pública, uma vez que as consequências para a saúde são muitas e variam do risco aumentado de morte prematura a graves doenças não letais, mas debilitantes, que afetam diretamente a qualidade de vida.

É comum a incidência de distúrbios fisiopatológicos causados pela obesidade, principalmente nas pessoas com IMC acima de $30 \mathrm{~kg} / \mathrm{m}^{2}$. Podem ser citados os distúrbios cardiovasculares (hipertensão arterial sistêmica, hipertrofia ventricular esquerda com ou sem insuficiência cardíaca, doença cerebrovascular, trombose venosa profunda, entre outros), distúrbios endócrinos (diabetes mellitus tipo II, dislipidemia, hipotireoidismo, infertilidade e outros), distúrbios respiratórios (apneia obstrutiva do sono, síndrome da hipoventilação, doença pulmonar restritiva).

O crescente aumento da obesidade, em especial quando o IMC $\geq 40,0 \mathrm{~kg} / \mathrm{m} 2$, e a sua associação com comorbidades influem diretamente o bem-estar físico, emocional e psicossocial, com impacto significativo sobre o declínio da QV.

Então, vendo a obesidade e o sobrepeso como problema de saúde pública, esta pesquisa objetivou identificar a percepção do efeito nos servidores públicos federais da sua participação no Projeto de Enfrentamento ao Sobrepeso e Obesidade, o qual foi desenvolvido pela DAS da SUPRIMIDO. Servidores ativos ou aposentados da SUPRIMIDO. com IMC $\geq 30$ ou 25 com comorbidades (Diabetes Melitus, Hipertensão Arterial, dislipidêmico, esteatose hepática, entre outras). Para calcular o Índice de Massa Corpórea - IMC: divide-se o peso $(\mathrm{Kg})$ pela altura $(\mathrm{m})$ ao quadrado.

O estudo mostrou que $70 \%$ dos participantes são do sexo feminino, a faixa etária variou de 21 a 70 anos, predominando, a faixa de 31 a 40 anos (35\%). Com relação ao nível de escolaridade, 50\% possuem o nível de graduação e $70 \%$ tem um tempo de atuação acima de 10 anos. Desse público, 90\% conseguem ver uma mudança evolutiva após a participação no projeto e considera ter um nível de saúde bom.

A avaliação da QV dos obesos e de pessoas com sobrepeso é de grande relevância. É importante que sejam realizados estudos que busquem, além de avaliar a QV desse público, a validação de escalas específicas para essa avaliação no Brasil. Esses estudos permitirão mais compreensão desse problema de saúde pública, análise do impacto da queda da QV na função diária desses indivíduos e serão parâmetros para comparar a eficácia de determinados tratamentos e programas voltados para esse fim.

Quanto ao limite do estudo, acredita-se que se faz necessário o levantamento do maior número de informações dos participantes antes de iniciar o cronograma do projeto, para que sejam vistas as evoluções dos participantes pós-projeto. Como exemplo das informações ausentes, são os dados antropométricos dos participantes do projeto de QV desenvolvido pela SUPRIMIDO., os quais não foram possíveis de serem identificados.

Então fica como sugestão de estudo comparar programas de outras instituições de ensino para verificar as reais necessidades de melhorias através de metodologias inovadoras, assim como realizar as medições antropométricas antes e depois da participação no projeto e analisar se o sobrepeso e obesidade são um problema apenas da QV ou é algo que reflete na QVT. 


\section{Referências}

Abravanel, F., Socha, T. C., Sell, I. M., Angela, L., \& Nesello, N. (2017). Eficácia de um programa de educação nutricional em grupo como estratégia para controle do sobrepeso e obesidade Effectiveness of a group nutrition education program as a strategy for overweight and obesity control, 12(4), 953-964. https://doi.org/10.12957/demetra.2017.28677

Adriana, J., Souza, M. A. De, \& De, R. A. B. (2017). Efeitos de um programa de exercícios em um adulto com acidente vascular encefálico após transplante cardíaco, 12(1), 24-33.

Brand, C., Schuh, L. X., \& Gaya, A. R. (2013). Atenção primária e os desafios à promoção da qualidade de vida de hipertensos Primary attention and challenges to improving the quality of life of hipertensive. Cinergis, 14(3), 161-165. https://online.unisc.br/seer/index.php/cinergis/article/viewFile/4000/3485

Campos, S. A. P., Lehnhart, E. dos R., Fossá, M. I. T., \& Balsan, L. A. G. (2017). Valores Relativos ao Trabalho em uma Instituição Pública de Ensino Superior. Revista Pretexto, 18(1), 11-27. https://doi.org/10.21714/pretexto.v18i1.2456

Carvalho, F. Q., Sampaio, D. A. (2010). A administração pública: Uma análise de sua história, conceitos e importância. https://www.webartigos.com/artigos/a-administracao-publica-uma-analise-de-sua-historia-conceitos-e-importancia/37923/.

Carvalho, M. et al., (2013). Produção Acadêmica em Recursos Humanos no Brasil. Revista de Administração de Empresas, 43(1), 105-122.

Coelho, R., et al. (2016). A saúde do trabalhador no âmbito universitário: Uma Parceria de Êxito na UFC. Revista de Psicologia, 7, 257-265

Corrêa, K., Gouvêa, G. R., \& Antonio, M. (2017). Qualidade de vida e características dos pacientes diabéticos Quality of life and characteristics of diabetic patients, pp. 921-930. https://doi.org/10.1590/1413-81232017223.24452015

Costa, S. D. S. (2014). Saúde bucal e sua influência na qualidade de vida do trabalhador: uma revisão de artigos publicados a partir do ano de 1990 Oral health and its influence on the quality of life of workers. 13(1), 2-12.

Dancey, C. P., \& Reidy, J. (2006). Estatística sem matemática para psicologia. Artmed.

Dias, C. L., \&Rozendo, K. C. T. (2015). O Contexto Laboral Acadêmico e as Possibilidades de Sofrimento Psíquico do Professor Universitário, 12, 13191327. https://doi.org/10.5747/ch.2015.v12.nesp.000754.

Gomes, D., \& Vieira, M. (2013). Valores organizacionais numa instituição pública de ensino do rn, 3, 77-89.

Guido, L. (2014). Stress e coping entre os servidores públicos de um hospital universitário, 180p. Tese de Doutorado. Escola de Enfermagem da Universidade de São Paulo, São Paudo, 2014.

Dias, P.C., Henrique, P., Anjos, L., A., Burlandy, L. (2017). Obesidade e políticas públicas: concepções e estratégias adotadas pelo governo brasileiro. Rio de Janeiro.

Grande, A. J., Botucatu, R., \& Clementino, V. (2013). Determinantes da qualidade de vida no trabalho: Ensaio Clínico Controlado e Randomizado por Clusters, 371-375.

Lasta, K. C., Gabriel, L., Ferrari, H. R., Luana, J., Dias, C. P., \& Tiggemann, C. L. (2018). Qualidade de vida de mulheres com diferentes faixas etárias de um programa de promoção à saúde, https://doi.org/http://dx.doi.org/10.22410/issn.2176-3070.v10i3a2018.1738

Maciel, N. M., Conti, M. H. S. De, Simeão, S. F. A. P., Corrente, J. E., Ruiz, T., \& Vitta, A. de. (2016). Morbidades referidas e qualidade de vida: estudo de base populacional. Fisioterapia e Pesquisa, 23(1), 91-97. https://doi.org/10.1590/1809-2950/14817923012016

Meirlles, N. Satisfação no Trabalho e fatores de estresse da equipe de enfermagem de um centro cirúrgico oncológico. Rev. Escola de Enfermgem Anna Nery., $1,78-88$.

Melo, P. B. De. (2016). Promoção e vigilância à saúde dos servidores públicos: a experiência da universidade federal do Ceará. Revista de Psicologia, 7, 151164.

Parise, J., \& Soler, Z. (2016). Qualidade de vida profissional de atendentes de central de telecomunicações, 69(4), 751-756.

Pasquali L. (2011). Psicometria: teoria dos testes na psicologia e na educação. (4a ed.), Vozes.

Pasternak, S. (2016). Habitação e saúde. https://doi.org/10.1590/S0103-40142016.00100004

Penteado, R., \& Gastaldello, L. (2015). Saúde e qualidade de vida de jornalistas, 29(2), 295-304.

Raiany, K., Silva, P., Fatima, M. De, Maia, D. M., Lafetá, J. C., Durães, G. M., \& Docente, B. (2018). Fatores associados ao bem-estar subjetivo em mulheres participantes de um programa social em Minas Gerais, 6(4), 419-423. https://doi.org/10.12662/2317-3076jhbs.v6i4.2111.p419-423.2018

Rueda, F., Serenini, A., \& Meireles, E. (2014). Relação entre qualidade de vida no trabalho e confiança do empregado na organização, 14(3), 303-314.

Silva, T., Aguiar, O. B. De, \& Fonseca, M. (2015). Associação entre sobrepeso, obesidade e transtornos mentais comuns em nutricionistas. https://doi.org/10.1590/0047-2085000000053

Sousa, J. E. De, \& Fernandes, R. (2014). Análise da qualidade de vida no trabalho e satisfação dos funcionários de uma empresa prestadora de serviços, 4 , 1035-1045. https://doi.org/10.7198/S2237-0722201400030004 
Research, Society and Development, v. 10, n. 13, e369101321277, 2021

(CC BY 4.0) | ISSN 2525-3409 | DOI: http://dx.doi.org/10.33448/rsd-v10i13.21277

Souza, E. (2015). Competitividade empresarial, gestão de pessoas e controle social: para pensar os dilemas da Qualidade de Vida no Trabalho (QVT), 5, 3548 .

Venturini, G., \& Ferreira, M. E. C. (2017). Repercussões da cirurgia bariátrica na qualidade de vida de pacientes com obesidade: uma revisão integrativa. Revista Brasileira de Obesidade, Nutrição e Emagrecimento, 12(November), 47-58. 